\title{
Influence of binder solvent on carbon-layer structure in electrical-double-layer capacitors
}

\author{
A BANERJEE, P SURESH KUMAR and A K SHUKLA* \\ Solid State and Structural Chemistry Unit, Indian Institute of Science, Bangalore 560 012, India \\ e-mail: akshukla2006@gmail.com
}

MS received 24 October 2012; revised 4 March 2013; accepted 22 March 2013

\begin{abstract}
Porous activated-carbons with a large surface-area have been the most common materials for electrical-double-layer capacitors (EDLCs). These carbons having a wide pore distribution ranges from micropores to macropores in conjunction with a random pore connection that facilitates the high specific-capacitance values. Pore distribution plays a central role in controlling the capacitance value of EDLCs, since electrolyte distribution inside the active material mainly depends on the pore distribution. This has a direct influence on the distribution of resistance and capacitance values within the electrode. As a result, preparation of electrodes remains a vital issue in realising high-performance EDLCs. Generally, carbon materials along with some binders are dispersed into a solvent and coated onto the current collectors. This study examines the role of binder solvents used for the carbon-ink preparation on the microstructure of the electrodes and the consequent performance of the EDLCs. It is observed that the physical properties of the binder solvent namely its dielectric constant, viscosity and boiling point have important role in determining the pore-size distribution as well as the microstructure of electrodes which influence their specific capacitance values.
\end{abstract}

Keywords. Electrical-double-layer capacitor; specific capacitance; electrodes microstructure; pore-size distribution; carbon inks.

\section{Introduction}

Electrical-double-layer capacitors (EDLCs) rely on non-faradaic electrostatic charging of the electrical double-layer formed at the electrode/electrolyte interface. EDLCs store electrical energy at the electrode/electrolyte interface through reversible ion adsorption onto the electrode surface, thus charging the electrical double-layer between an electronic conductor and a liquid ionic-conductor, namely the electrolyte, through a non-faradaic contribution. The few nano-meter thick electrical double-layers at the phase boundary between the electrode and electrolyte can be referred to as a nano-dielectric akin to a parallel-plate capacitor wherein the current collector of each electrode contacts high-surface-area conductive material, with wide pore-distribution ranges, impregnated with an electrolyte.

Activated carbons have attracted much attention as materials in symmetrical EDLCs due to their very high surface-area, wide pore-size-distribution, low cost, and broad potential window. In aqueous solution, they can

*For correspondence be operated between 0 and $1.2 \mathrm{~V}$, while in ionic liquid they have been used in the potential range of 0 $3.5 \mathrm{~V}$. Since the performance of double-layer capacitors merely depends on the electrochemically-active-surface area, the approach of electrolyte towards the carbon surface is critical. Accordingly, a carbon with high surfacearea alone does not meet the necessary condition, as an appropriate pore-size distribution is equally desirable. ${ }^{1}$

As reported in the literature ${ }^{2-5}$, the solvent used for the preparation of catalyst ink plays an important role on the microstructure and pore-size distribution of electrode catalyst layers used for fuel cells. Solvents with low dielectric constant are shown to be more favoured as the catalyst is dispersed well in these solvents. Furthermore, the pore-size distribution is better with the solvents of low polarity, and is reflected in the high performance of the fuel cell. It is also reported that high viscosity of the solvents is preferred for a stable catalyst suspension during the ink deposition process. Simultaneous heating during the ink preparation was shown to produce more porous coating. Even though it is established that the physical properties of solvents used for ink preparation influence the active surface area and pore-size distribution of the electrodes used for fuel cells, similar studies on supercapacitor electrodes are lacking in the literature. It is noteworthy that while in 
fuel cells faradaic reactions occur, in EDLC electrodes, only non-faradaic reactions like adsorption/desorption of ions take place. Consequently, the active surface area in EDLCs has a different perspective as compared to fuel cell electrodes, and has not been addressed in the literature.

In this study, we report our experimental findings on the performance of carbon-based EDLCs employing carbon electrodes fabricated by using various organic solvents for carbon ink preparation. Meadwestvaco carbon X-090177 (85 wt.\%) and activated charcoal (10 wt.\%) are used as active material and additive, respectively, along with Poly (vinylidene fluoride) (PVDF) binder (5 wt.\%) to prepare the carbon ink using various organic solvents. $1.2 \mathrm{~V}$ EDLC single cells are performance tested using electrochemical tools. The data are analysed invoking pore-size distribution, internal resistance and specific capacitance values of the prototype cells fabricated using carbon electrodes. It is surmised that the solvent does play an important role on the performance of the EDLCs.

\section{Experimental}

\subsection{Physicochemical characterization of Meadwestvaco carbon X-090177 and activated charcoal}

Meadwestvaco carbon X-090177 and activated charcoal AR (S D Fine-Chem Limited, Mumbai, India) were characterized by powder X-ray diffraction (PXRD), scanning electron microscopy (SEM) and particlesize distribution from dynamic light scattering (DLS) analysis. X-ray diffraction pattern for the carbon samples were recorded on a Philips X'pert-Pro $\mathrm{X}$-ray Diffractometer equipped with X'cellerator detector at scan speed $\left({ }^{\circ} / \mathrm{s}\right)$, step size $\left(^{\circ}\right)$ and time per step (s) values of $0.035,0.016$ and 30 , respectively. SEM images of carbons were obtained on JEOL JSM5600LV Scanning Electron Microscope and samples were drop casted on silicon wafer. Particle-size distribution for the carbon samples were obtained from water dispersion (carbon concentration $=0.5 \mathrm{mg} \mathrm{ml}^{-1}$ ) by Malvern Particle-Size Analyser. Pore-size distribution of carbon coatings on the graphite sheet were recorded on a Capillary Flow Porometer, CFP-1500-AEXBB Model-D, from Porous Materials, Inc (PMI).

\subsection{Preparation and characterization of carbon electrodes}

5 wt.\% of Poly (vinylidene fluoride) (PVDF) polymer was dissolved in required quantity in one of the solvents, namely triethylphosphate (TEP), trimethylphosphate (TMP), tetramethylurea (TMU), dimethyl formamide (DMF), dimethyl acetamide (DMA), hexamethylphosphoramide (HMPA), Nmethyl-2-pyrrolidone (NMP), dimethyl sulphoxide (DMSO) or propylene carbonate (PC), by ultrasonication. Subsequently, 85 wt.\% of Meadwestvaco carbon X-090177 and 10 wt.\% of activated charcoal were dispersed into the PVDF solution to obtain a thick carbon-ink. It is noteworthy that several high surface area activated carbon samples, namely Meadwestvaco carbons (X-090177, X-090185, X-090189, X-090120), energ2 carbons (V2-12, P2-15), Vulcan carbons (XC-72, XC-72R, XC-500), Ketjen Black, CDX-975, Black Pearls-2000, were screened and among these Meadwestvaco carbon X-090177 was found to exhibit highest capacitance value. The resulting ink was applied onto $0.3 \mathrm{~mm}$ thin graphite sheets of size $4.5 \mathrm{~cm} \times 7 \mathrm{~cm}$ with a tag of dimensions: $1 \mathrm{~cm} \times 1.5 \mathrm{~cm} \times 0.3 \mathrm{~mm}$. Weight of graphite current collector was $3 \mathrm{~g}$. The graphite electrodes were subsequently dried in an air oven at $80^{\circ} \mathrm{C}$. Weight of the electrodes was taken up to three constant readings. Each side of the electrode contained $\sim 0.5 \mathrm{~g}$ carbon with a thickness of $\sim 300 \mu \mathrm{m}$ observed by cross-sectional SEM image (see figure S1 in supplementary information). The dried electrodes were used for physical characterization as also for assembling the prototype cells.

\subsection{Assembly and characterization of $1.2 \mathrm{~V} \mathrm{EDLC}$ prototype cells}

Symmetric electrical double-layer capacitors were assembled with two carbon electrodes separated by $1 \mathrm{~mm}$ thick polyethylene mesh separator. All the experiments were carried out in aq. $6 \mathrm{M}$ sulphuric acid as electrolyte. An Autolab Potentiostat/GalvanostatModel 30 was used for electrochemical characterization of the prototype cells by cyclic voltammetry, a.c. impedance spectroscopy and constant-current chargedischarge studies. All measurements were carried out at room temperature $\left(\sim 28^{\circ} \mathrm{C}\right)$.

\section{Results and discussion}

\subsection{Physicochemical properties of Meadwestvaco carbon X-090177 and activated charcoal}

X-ray diffraction patterns for Meadwestvaco carbon X-090177 and activated charcoal are shown in figure 1a and $b$, respectively. The XRD plots of both samples do not exhibit any sharp peaks, except a broad peak around 


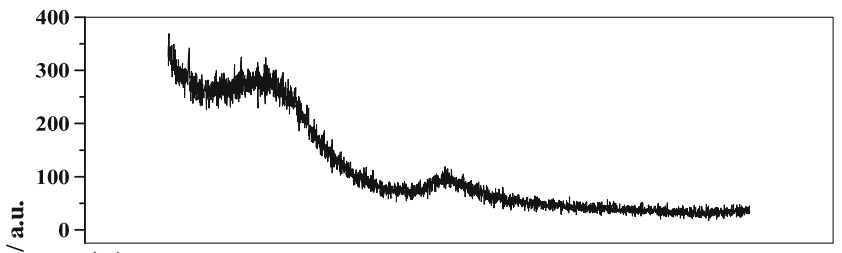

(a)

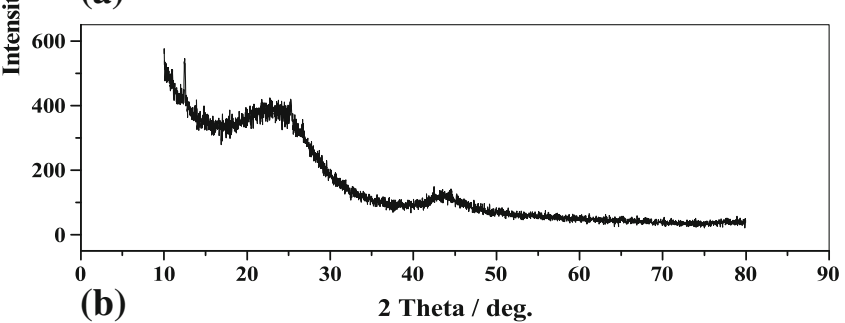

Figure 1. XRD patterns for (a) Meadwestvaco carbon X-090177 and (b) activated charcoal.

$2 \theta=22^{\circ}$, indicating the highly amorphous nature of the samples. Further analysis of the samples using SEM provides information about the morphology and particle size as depicted in figure 2. It is observed from the images that most of the particles are in the range of 4-8 $\mu \mathrm{m}$ for the Meadwestvaco carbon X-090177, while activated charcoal shows particle size $<4 \mu \mathrm{m}$. These values are in agreement with the particle size distribution obtained by dynamic light scattering (DLS) measurements as presented in figure 3. Form the DLS studies, we have obtained the $\mathrm{d}_{90}, \mathrm{~d}_{50}, \mathrm{~d}_{10}, \mathrm{D}[4,3]$, D[3,2] values of Meadwestvaco carbon X-090177 as $4.42,7.83,12.24,8.13,6.82 \mu \mathrm{m}$, respectively, while for the activated charcoal, the respective values are 0.09 , 2.86, 13.48, 5.38, $0.34 \mu \mathrm{m}$.

The surface area of active materials affects the capacitance of EDLCs as these two parameters are directly related. We have measured the surface area of both Meadwestvaco carbon X-090177 and activated charcoal using nitrogen adsorption/desorption isotherm.

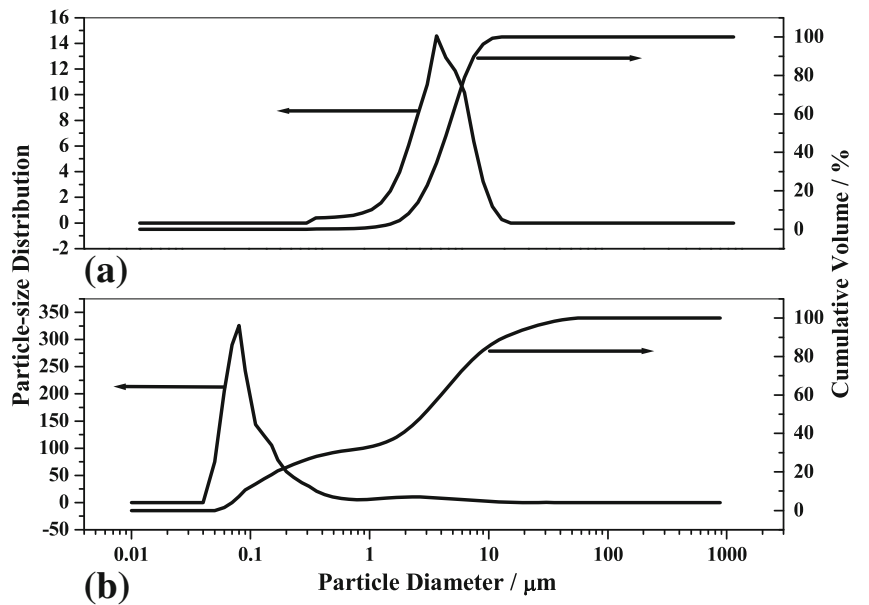

Figure 3. Particle size distribution obtained by dynamic light scattering method for (a) Meadwestvaco carbon $\mathrm{X}-090177$ and (b) activated charcoal.

The isotherms, as shown in figure S2 ( $a$ and $b$ ) in supplementary information, are type I isotherm with negligible $\mathrm{H} 4$ hysteresis. ${ }^{6}$ The surface area of microporous Meadwestvaco carbon X-090177 and activated charcoal are found to be 1512 and $974 \mathrm{~m}^{2} \mathrm{~g}^{-1}$ with average pore-size of 1.89 and $1.96 \mathrm{~nm}$, respectively. Further analysis reflects that carbon does not contain any additional functional groups (see supplementary information figures S3, S4 and S5).

\subsection{Selection of solvents for carbon ink}

In order to investigate the effect of solvent on the carbon ink composition and consequently on the active carbon layer microstructure, a series of solvents was utilized. PVDF solubility is the central point in choosing the solvent. A detailed study on the solubility of PVDF had been reported by Bottino et al. ${ }^{7}$ Among 46 solvents used by Bottino et al. only 8 solvents were found

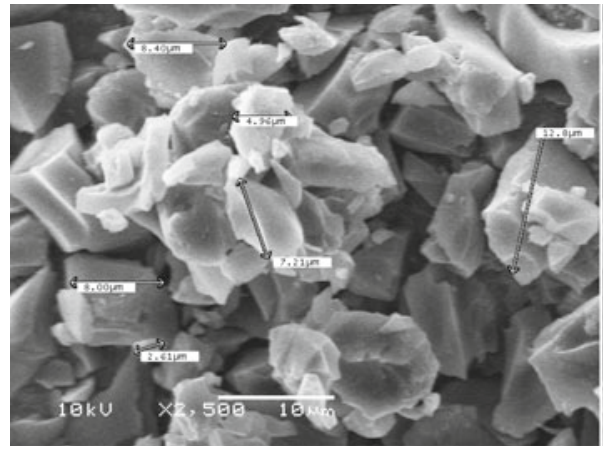

(a)

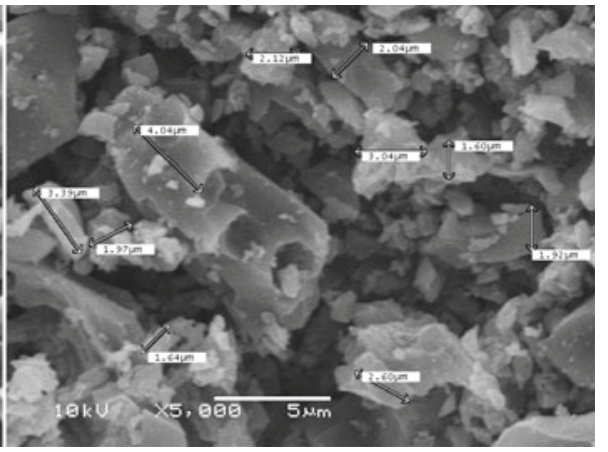

(b)

Figure 2. Scanning electron microscopic images for (a), Meadwestvaco carbon X-090177 and (b) activated charcoal. 
to be good solvents for PVDF. Apart from these 8 solvents, we had also tried Propylene carbonate $(\mathrm{PC})$ in the present study as our experience had shown it to be an equally good solvent. The degree of PVDF solubility in various solvents is depicted in the supporting information (figure S6). Physical properties like boiling point, dielectric constant and viscosity of all the solvents used in this study are listed in the supporting information (table S1).

The surface structure of EDLC electrodes prepared using the aforementioned solvents was studied by SEM images as shown in figure 4 . The pore-size distribution of the carbon layer of these electrodes was measured and is plotted in figure 5. These data clearly indicate
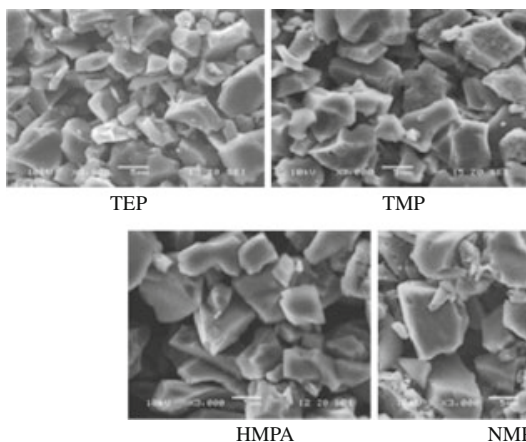

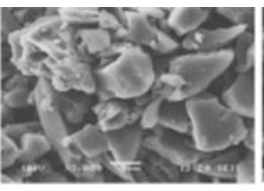

TMU

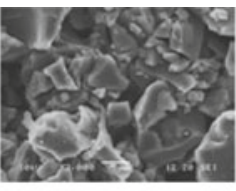

DMF

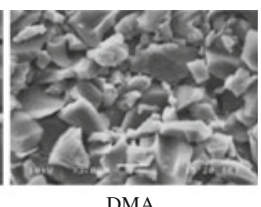

DMA
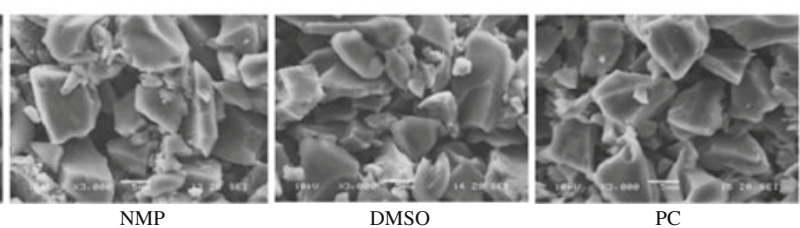

Figure 4. Scanning electron microscopic images for different EDLC electrodes made by different solvents.
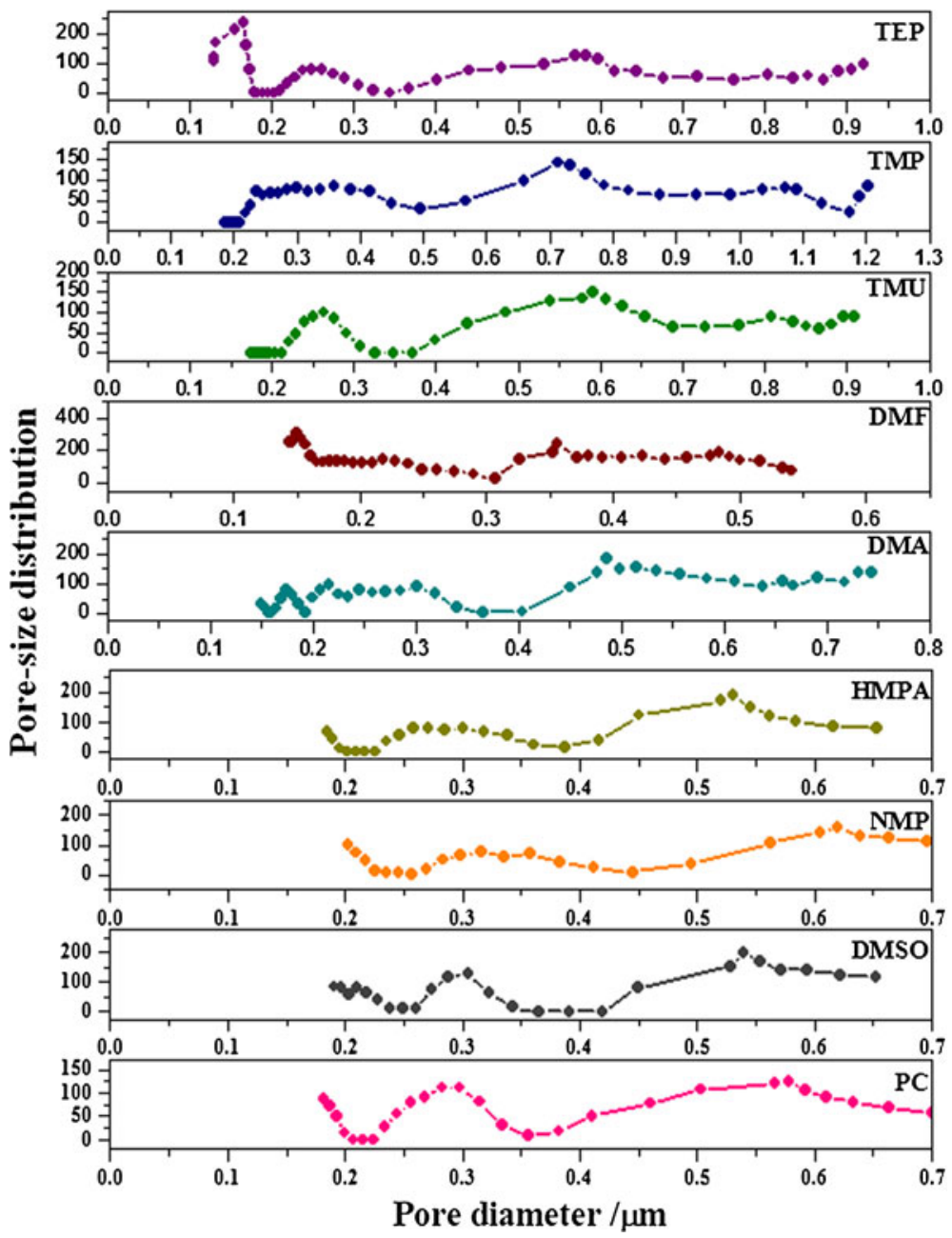

Figure 5. Pore size distribution of EDLC electrodes, where carbon inks were prepared by using different solvents. 
that the microstructure of carbon layer of EDLC electrodes is highly influenced by the physical properties of solvents used in the ink preparation and have varying effects on the microstructure due to their differing dielectric constant values, boiling point and viscosity. We have earlier confirmed that both the carbon samples are free from surface functionalities and other impurities to any appreciable amount. As a result, a good dispersion of ink is obtained when solvents with lower dielectric constant are employed. High viscosity helps maintaining a stable suspension during the ink deposition. The fast solvent removal during the ink deposition process is highly appreciated for homogeneous distribution of the particles as well as pores. ${ }^{3}$ This indicates that solvents with lower dielectric constant, higher viscosity and lower boiling point are preferable for carbon ink preparation.
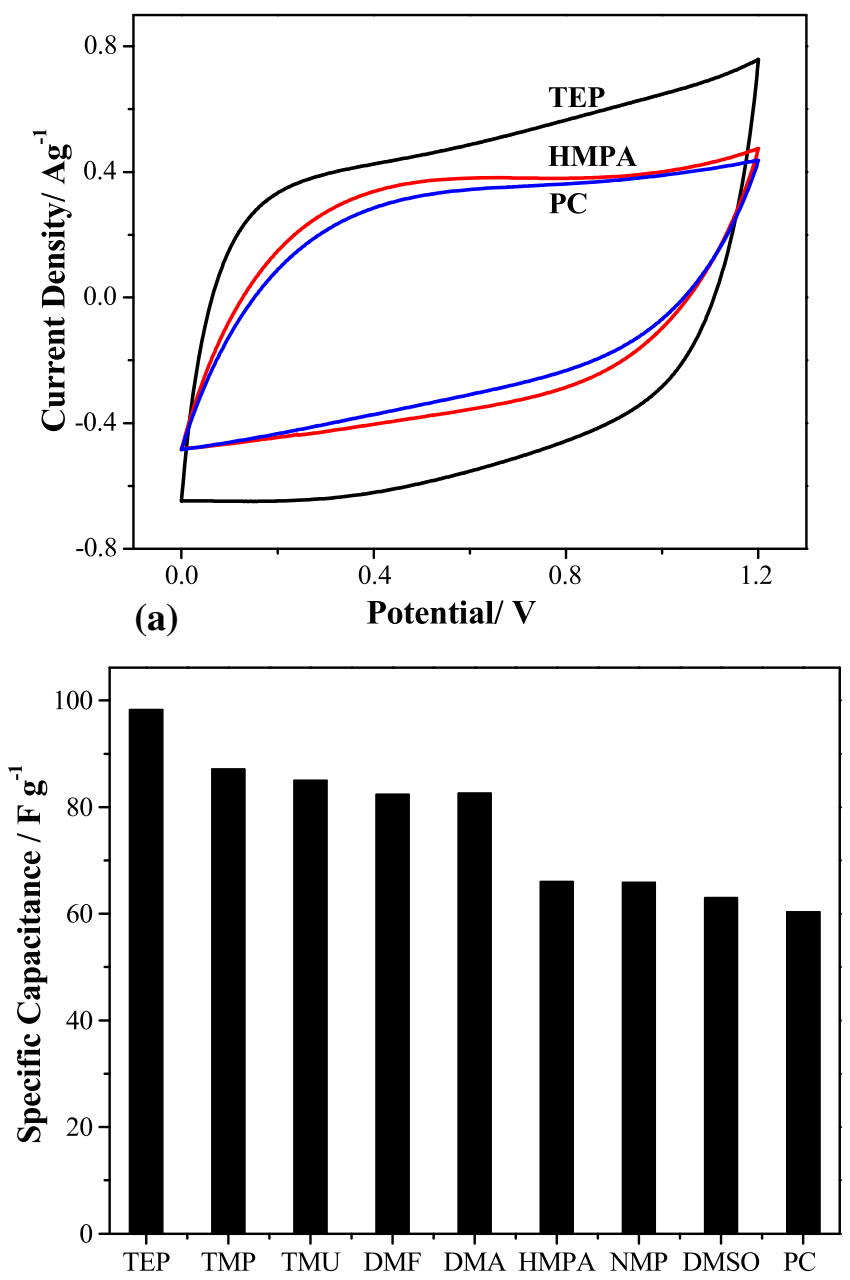

(b)

Figure 6. (a) Cyclic voltammogram of EDLCs, where carbon inks were prepared by different solvents. (b) Specific capacitance values of the prototype cells calculated from cyclic voltammogram of EDLCs.

\subsection{Electrochemical characterization of $1.2 \mathrm{~V} \mathrm{EDLCs}$}

3.3a Cyclic voltammetry of $1.2 \mathrm{~V}$ EDLCs: Cyclic voltammetry $(\mathrm{CV})$ is a widely used tool for the analysis of EDLCs and a perfect double layer capacitor is modelled to be having a rectangular voltammogram. We had measured the $\mathrm{CV}$ of the prototype cells with electrodes that were prepared using the aforementioned solvents. The CV studies at a scan rate of $10 \mathrm{mVs}^{-1}$ were carried out in $6 \mathrm{M}$ aq. $\mathrm{H}_{2} \mathrm{SO}_{4}$, as it happens to be the optimized concentration. $^{8}$

Representative CVs for the prototype cells are shown in figure 6a. All the electrodes exhibit almost rectangular cyclic voltammograms indicating the absence of any faradaic process and the presence of only doublelayer charging/discharging. The specific capacitance values calculated from these cyclic voltammograms are presented in figure 6b. It is clear from these data that the solvents affect the performance of the cell substantially; for example, electrodes fabricated using TEP exhibit maximum, while electrodes using PC yield lowest specific capacitance value. The specific capacitance values at varying current densities are obtained by the charge/discharge technique as described in the following section.

3.3b Internal resistance of $1.2 \mathrm{VEDLCs:}$ For obtaining higher performance, internal resistance values for the EDLC cells should be as low as possible. We had measured the internal resistance values of these prototype cells by A. C. impedance spectroscopy ${ }^{1}$, by taking the $\mathrm{X}$-intercept value at high-frequency region in the Nyquist plot. The measured data are presented in figure 7. The variation in the resistance values can be explained by the solvent properties. Solvents with low

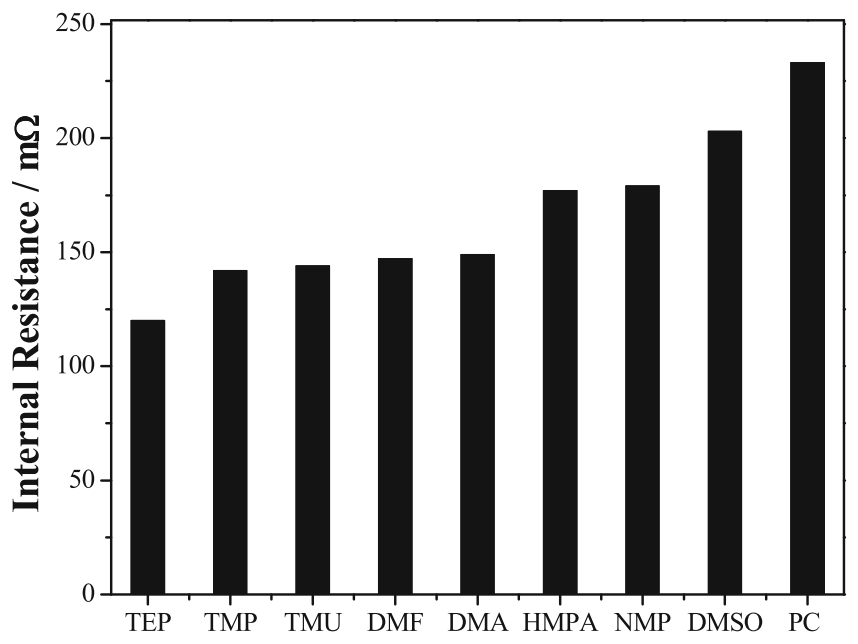

Figure 7. Internal resistance values of EDLCs measured from Nyquist plots of A. C. impedance spectroscopy. 
dielectric constant lead to low internal resistance for the prototype cells. Homogeneously distributed finer particles and smaller pores are responsible for lower internal resistance values of EDLCs since they facilitate many electron transfer pathways within the electrode structure that are interconnected.

\section{$3.3 \mathrm{c}$ Charge/discharge characteristics of $1.2 \mathrm{~V}$} EDLCs: Measurements on electrochemical double layer capacitance values are possible by constant current charge/discharge technique. In the present study, galvanostatic charge/discharge cycles were carried

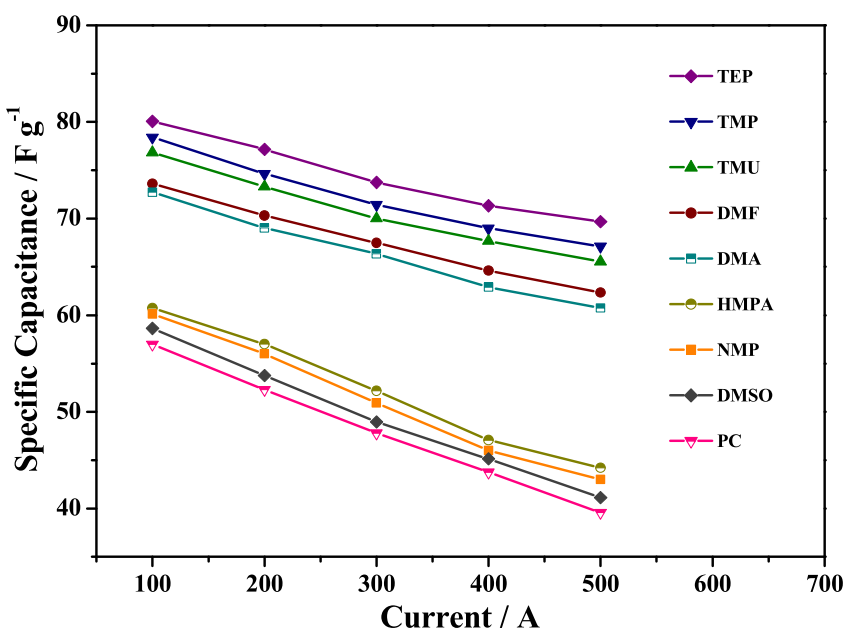

Figure 8. Variation of specific capacitance with current for EDLCs, where carbon inks were prepared by using different solvents. out at varying current loads between $100 \mathrm{~mA}$ and $500 \mathrm{~mA}$. The capacitance values are calculated from the discharge data and are presented in figure 8 for all the solvents used for the carbon ink preparation. The plots represent two sets of values, one set of high capacitance and the other set of lower capacitance. It is observed that the high capacitance values are obtained when low dielectric constant solvents were used for the ink preparation. When solvents with similar dielectric constants were used, the capacitance values are determined by other physical properties like boiling point and viscosity. For example, TEP has lowest dielectric constant but shows highest capacitance, while PC has highest dielectric constant but exhibits lowest capacitance values. By contrast, DMF and NMP have similar dielectric constant values albeit the capacitance values are quite different. It is noteworthy that the boiling point and viscosity are much higher for NMP than DMF.

A general behaviour of the relationship between capacitance values and the physical properties of the solvent used for the ink preparation is presented in figure 9. It is obvious from the plots that the dielectric constant of the solvent makes a major contribution towards the performance of the single cell capacitors. When the dielectric constant values are very close, the boiling point as well as the viscosity of the solvents affect the capacitance values. These findings are in agreement with the results obtained for the solvent effect on ink preparation of catalyst materials used in fuel cell. ${ }^{2-5}$ Even though a quantitative relationship between the solvent properties and the performance

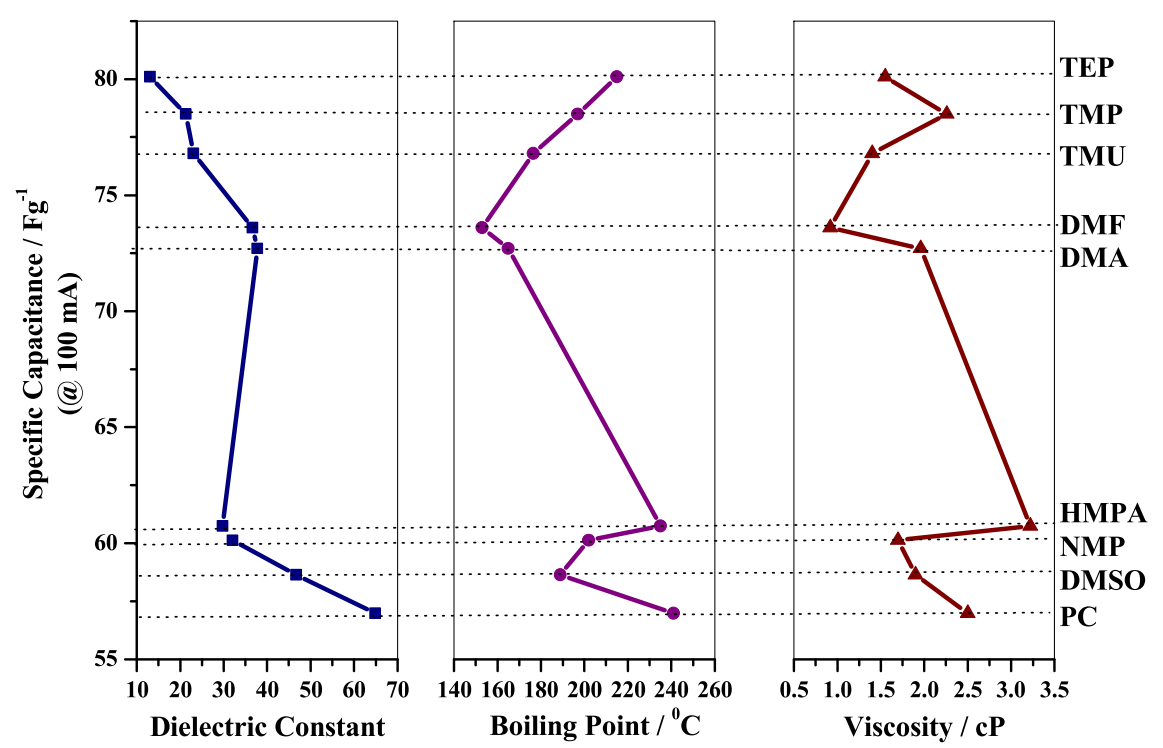

Figure 9. Variation of specific capacitance with the dielectric constant ( $\mathbf{\square})$, boiling point $(\boldsymbol{O})$ and viscosity $(\boldsymbol{\Lambda})$ of the solvent used for the carbon ink preparation. 
could not be reached in the present study, it is clear that lower dielectric constant, boiling point and high viscosity are the favoured parameters for high performance carbon-based EDLCs.

\section{Conclusions}

A series of EDLC electrodes have been fabricated from carbon ink using a variety of organic solvents. It is observed that the microstructure of carbon layers is highly sensitive to physical properties of solvents such as dielectric constant, boiling point and viscosity. When low dielectric constant solvents are used for the ink preparation, homogenously distributed finer particles and smaller pores are obtained which leads to the higher capacitance of EDLCs. Findings from the present studies are important for a better understanding of the role of solvent during ink preparation on the performance. However, further studies are required to establish a quantitative relationship between the solvent physical properties and the double layer capacitance.

\section{Supporting material}

For supporting information figures S1-S6 and table S1, see the website (www.ias.ac.in/chemsci).

\section{Acknowledgements}

Financial support from the Department of Science and Technology (DST), Government of India and Indian Institute of Science (IISc), Bangalore under its Energy Storage Systems Initiative (ESSI) are gratefully acknowledged. AB thanks the Council of Scientific and Industrial Research (CSIR), New Delhi for a Senior Research Fellowship.

\section{References}

1. Conway B E 1999 Electrochemical supercapacitors: Scientific fundamentals and technological applications (New York: Kluwer-Plenum)

2. Kim J H, Ha H Y, Oh I H, Hong S A and Lee H I 2004 J. Power Sources 13529

3. Fernandez R, Ferreira-Aparicio P, daza L 2005 J. Power Sources $\mathbf{1 5 1} 18$

4. Sung K A, Jung H Y, Kim W K, Cho K. Y and Park J K 2007 J. Power Sources 169271

5. Chisaka M and Daiguji H 2009 J. Electrochem. Soc. 156 B22

6. Sing S W, Everett D H, Haul R A W, Moscou L, Pierotti R A, Rouquerol J and Siemieniewska T 1985 IUPAC Recommendations 1984: Pure Appl. Chem. 57603

7. Bottino A, Capannelli G, Munari S and Turturro A 1988 J. Polym. Sci. Part B: Polym. Phys. 26786

8. Darling H. E 1964 J. Chem. Eng. Data 9421 